\title{
Blood Vessel-Derived Acellular Matrix for Vascular Graft Application
}

\author{
Luigi Dall'Olmo, ${ }^{1}$ Ilenia Zanusso, ${ }^{2}$ Rosa Di Liddo, ${ }^{2}$ Tatiana Chioato, ${ }^{2}$ \\ Thomas Bertalot, ${ }^{2}$ Enrica Guidi, ${ }^{2}$ and Maria Teresa Conconi ${ }^{2}$ \\ ${ }^{1}$ Istituto Oncologico Veneto, 35131 Padua, Italy \\ ${ }^{2}$ Department of Pharmaceutical and Pharmacological Sciences, University of Padua, Via Marzolo 5, 35131 Padua, Italy
}

Correspondence should be addressed to Maria Teresa Conconi; mariateresa.conconi@unipd.it

Received 21 March 2014; Revised 12 June 2014; Accepted 7 July 2014; Published 16 July 2014

Academic Editor: Luca Urbani

Copyright (C) 2014 Luigi Dall'Olmo et al. This is an open access article distributed under the Creative Commons Attribution License, which permits unrestricted use, distribution, and reproduction in any medium, provided the original work is properly cited.

\begin{abstract}
To overcome the issues connected to the use of autologous vascular grafts and artificial materials for reconstruction of small diameter $(<6 \mathrm{~mm})$ blood vessels, this study aimed to develop acellular matrix- (AM-) based vascular grafts. Rat iliac arteries were decellularized by a detergent-enzymatic treatment, whereas endothelial cells (ECs) were obtained through enzymatic digestion of rat skin followed by immunomagnetic separation of CD31-positive cells. Sixteen female Lewis rats (8 weeks old) received only AM or previously in vitro reendothelialized AM as abdominal aorta interposition grafts (about $1 \mathrm{~cm})$. The detergent-enzymatic treatment completely removed the cellular part of vessels and both MHC class I and class II antigens. One month after surgery, the luminal surface of implanted AMs was partially covered by ECs and several platelets adhered in the areas lacking cell coverage. Intimal hyperplasia, already detected after 1 month, increased at 3 months. On the contrary, all grafts composed by AM and ECs were completely covered at 1 month and their structure was similar to that of native vessels at 3 months. Taken together, our findings show that prostheses composed of AM preseeded with ECs could be a promising approach for the replacement of blood vessels.
\end{abstract}

\section{Introduction}

Cardiovascular diseases (CVDs) represent the leading cause of death in the western countries [1]. Since pharmacological treatment mainly consisting in antiplatelet drugs and cholesterol-lowering agents (statins) has been proven to be often not sufficient [2], the implantation of a vascular graft may be needed. Although autologous vessels, such as the internal mammary artery, radial artery, or the saphenous vein, are considered the golden standard for replacement of malfunctioning or diseased blood vessels, their availability is limited especially in elderly patients [3]. Synthetic materials, such as Dacron and expanded polytetrafluoroethylene (ePTFE), perform reasonably in high-flow, low-resistance conditions and they can be used successfully to substitute large diameter vessels [4]. Nevertheless, they are not as suitable for small diameter $(<6 \mathrm{~mm})$ arterial grafts due to their thrombogenicity [5]. Thus, the development of an effective vascular graft, possessing biomechanical properties matching those of native vessel, has become one of the main targets of tissue engineering.

Three approaches have been designed for vascular regeneration: (i) biodegradable synthetic polymer-based constructs, (ii) cell self-assembly blood vessels, and (iii) decellularized tissue grafts.

Several polymers have been used to obtain tissueengineered blood vessels (TEBVs): poly(dimethylsiloxane), poly(caprolactone), poly(methyl methacrylate), poly-L-lactic acid (PLLA), polyglycolic acid, poly(glycerol sebacate), and polyvinyl alcohol (PVA) [6, 7]. Notably, Shinoka et al. [8] carried out the first human clinical study enrolling 42 patients who underwent cavopulmonary connection with a TEBV composed of a copolymer of $\varepsilon$-polycaprolactone-polylactic acid reinforced with woven polyglycolic acid previously seeded with autologous bone marrow-derived mononuclear cells. Although some grafts presented stenosis, long-term results at 2 years indicated that TEBV functioned well without any aneurysm or graft rupture. 
Another approach for the fabrication of TEBVs is cell self-assembly developed by L'Heureux and colleagues [9] who used entirely autologous cells cultured in vitro. Sheets of smooth muscular cells (SMCs) and fibroblasts were grown to overconfluence and then assembled over a mandrel to form a tubular structure that was cultured for 6-8 weeks. During this incubation period, the autologous cells arranged themselves circumferentially producing large amounts of extracellular matrix (ECM). Recently, promising results were obtained after implantation of these TEBVs into 10 patients receiving hemodialysis with failed arteriovenous fistulas [10, 11]. Although self-assembly grafts possess excellent mechanical properties and nonimmunogenic nature, a very long culture period (about $8-10$ weeks) is required to obtain an implantable construct.

Finally, decellularized tissues, named also acellular matrices (AMs), can be obtained from various anatomical sites through several procedures involving both physical and chemical agents, such as surfactants and enzymes [12]. These biomaterials possess a preformed structurally organized ECM containing angiogenic growth factors, such as bFGF and VEGF $[13,14]$, and lacking immunogenic issues. Small intestinal submucosa (SIS), porcine carotid artery, aorta, and even canine carotid arteries have been evaluated using animal models $[15,16]$. Beside the potential risk of viral transmission from animal tissue, the implantation of decellularized xenografts gave disappointing results. Indeed, the treatment of patients with decellularized bovine ureters resulted in high failure rate probably due to the presence of residual immunogenic contaminants, such as galactosealpha-1,3-galactose (alpha gal) [17]. Thus, the use of homologous AMs seems to be a more suitable approach for vascular replacement.

Overall, the major drawbacks connected to described TEBV s are thrombogenicity, the occurrence of intimal hyperplasia, the progressive atherosclerotic degeneration, and the time required for culturing the cells. Starting from these considerations, the present study developed AM-based vascular grafts composed of AMs derived from iliac arteries and skin microvascular ECs and evaluated their effectiveness as abdominal aorta interposition grafts in Lewis rats.

\section{Materials and Methods}

2.1. Materials. Phosphate-buffered saline (PBS) tablets were purchased from Gibco Invitrogen Corp. (Paisley, UK). Rabbit monoclonal anti-MHC I and II and rabbit polyclonal antivon Willebrand factor primary antibodies were provided by Abcam (Cambridge, UK). Horse pan-specific secondary antibody, DAB Peroxidase Substrate, Fluorescein Avidin DCS, and Vectashield Mounting Medium were from Vector Laboratories (Burlingame, CA, USA). Collagenase B and Dispase II were obtained from Roche Applied Science (Indianapolis, IN, USA). The Endothelial Cell Growth Medium MV2 was purchased from PromoCell GmbH (Heidelberg, Germany). Cell strainer, tissue culture-treated dishes, and fibronectin were from BD Biosciences (San Jose, CA, USA). Mouse monoclonal anti-rat-CD31 antibody was provided by Millipore (Billerica, MA, USA). Dynabeads M-450 were obtained from
Life Technologies (Monza, Italia). Movat pentachromic stain kit was from Diapath S.p.A. (Martinengo, Italy). Contramal was purchased by Grünenthal (Aachen, Germany), whereas Terramicina was from Phibro Animal Health Corporation (Teaneck, NJ, USA). All other chemicals and reagents were provided by Sigma-Aldrich (St. Louis, MO, USA).

2.2. Animals. All procedures described and animal protocols were approved by the Institutional Animal Care Committee of the University of Padua and by the Italian Health Department. Lewis rats ( 8 weeks old, 200-300 g body weight) were purchased from Charles-River (Como, Italy). Male animals were sacrificed using $\mathrm{CO}_{2}$ inhalation and iliac arteries as well as dermis were collected and rinsed with PBS. In vivo experiments were carried out on female rats.

2.3. Acellular Matrices. AMs were prepared by Meezan et al. method [18] with minor modifications. Briefly, iliac arteries were processed with distilled water for $72 \mathrm{~h}$ at $4^{\circ} \mathrm{C}, 4 \%$ sodium deoxycholate for $4 \mathrm{~h}$, and 2,000 kU deoxyribonuclease I (DNase-I) in $1 \mathrm{M} \mathrm{NaCl}$ for $3 \mathrm{~h}$. The treatment was repeated twice till the cells were completely removed. The endothelium was detached incubating the vessels with collagenase IV $(0.05 \%$ in $\mathrm{PBS})$ at $37^{\circ} \mathrm{C}$ for $1 \mathrm{~min}$. To verify the lack of cells, AMs were fixed with $10 \%$ formalin in PBS, paraffinembedded, and stained with hematoxylin/eosin (H/E). On the other hand, the absence of cellular membrane residuals was evaluated by immunoistochemistry. Briefly, slices were treated with hydrogen peroxide for $30 \mathrm{~min}$ at room temperature (RT) and nonspecific binding sites were blocked with $10 \%$ bovine serum albumin (BSA) in PBS for $20 \mathrm{~min}$ at RT. Samples were incubated for $60 \mathrm{~min}$ with rabbit monoclonal anti-MHC I and II primary antibodies (1:500 in 4\% BSA in PBS) and then with the horse pan-specific secondary antibody (1:8 in 4\% BSA in PBS) for $30 \mathrm{~min}$ at RT. The reaction was developed with DAB Peroxidase Substrate following the manufacturer's instruction. Finally, nuclei were counterstained with hematoxylin. Negative controls were obtained by omitting the primary antibody. Alternatively, AMs were fixed with $4 \%$ glutaraldehyde in $0.1 \mathrm{M}$ cacodylate buffer ( $\mathrm{pH} 7.2$ ) for $24 \mathrm{~h}$ and dehydrated with $70 \%$ and $90 \%$ ethanol ( $2 \mathrm{~h}$ each) and 100\% ethanol overnight. After critical point drying and gold sputtering, samples were examined by a scanning electron microscope (SEM; Stereoscan-205 S, Cambridge, UK) using a standard protocol.

2.4. Cell Cultures. Dermis, obtained from the abdomen of male rats, was rinsed in PBS, minced, and treated with $0.25 \%$ Collagenase B and $0.25 \%$ Dispase II for $1 \mathrm{~h}$ at $37^{\circ} \mathrm{C}$. The digested tissue was filtered through a $100 \mu \mathrm{m}$ cell strainer. Cell suspension was centrifuged and resuspended in culture medium MV2. Cells were then seeded on fibronectin$\left(1 \mu \mathrm{g} / \mathrm{cm}^{2}\right)$ coated dishes and cultured at $37^{\circ} \mathrm{C}$ with $5 \% \mathrm{CO}_{2}$. To obtain pure skin microvascular ECs, cultures grown to $80 \%$ confluence were immunoseparated using Dynabeads M-450 previously coated with the mouse monoclonal antirat-CD31 antibody following manufacturer's instruction [19]. Briefly, cells were incubated with magnetic beads for $30 \mathrm{~min}$ 
at $4^{\circ} \mathrm{C}$ ( 5 beads/cell). ECs bound to the coated beads were collected with a magnetic particle concentrator and unbounded cells were removed by means of 2 washes with culture medium. Finally, ECs were seeded on fibronectincoated dishes, cultured with MV2 medium, and used until the 4 th passage. The isolated cells were characterized by immunofluorescence performing with rabbit polyclonal antivon Willebrand factor $(1: 400)$. Briefly, cells were fixed with $4 \%$ formalin for $10 \mathrm{~min}$ at $4^{\circ} \mathrm{C}$, washed in PBS, and incubated at RT for $1 \mathrm{~h}$ with $10 \%$ horse serum in PBS and, for $1 \mathrm{~h}$, with the primary antibody. After rinsing with PBS, cells were treated with the horse pan-specific secondary antibody (1:8) for 30 min and with Fluorescein Avidin DCS 1: 500 in HEPES $10 \mathrm{mM}$ and $\mathrm{NaCl} 0.15 \mathrm{M}$ for $10 \mathrm{~min}$. Samples were mounted with mounting medium with DAPI.

2.5. Cultures of ECs on AMs. Under static conditions, ECs $\left(4 \times 10^{5} / \mathrm{cm}^{2}\right)$ were seeded onto the luminal surface of AMs, previously incubated with MV2 medium for $3 \mathrm{~h}$ at $37^{\circ} \mathrm{C}$. Cultures were maintained for $72 \mathrm{~h}$ in MV2 medium and then fixed for morphological analysis or in vivo implanted.

2.6. In Vivo Experiments. Female Lewis rats (8 weeks old) were divided into two groups according to the kind of the implanted graft: group 1AMs $(n=7)$ and group 2 AMs plus ECs $(n=9)$. Under isoflurane anesthesia (3\% isoflurane carried by oxygen, $1 \mathrm{~L} / \mathrm{min}$ ), the abdominal area was shaved and aseptically prepared using povidone-iodine (Betadine). The muscles were exposed with a $3 \mathrm{~cm}$ abdominal incision and, after peritoneal incision, animals received analgesic ( $5 \mathrm{mg} / \mathrm{Kg}$ Tramadol, Contramal) intraperitoneally. The abdominal aorta was exposed and isolated and, after clamping the vessel, a segment of aorta was excised and the graft (about $1 \mathrm{~cm}$ in length) was anastomosed proximally and distally end-to-end using continuous 10.0 polypropylene sutures. Animals received antibiotic (Terramicina, $60 \mathrm{mg} / \mathrm{kg}$ ) on the 3rd and 6th days after surgery and Contramal for 3 days postoperatively. No anticoagulants or antiplatelets were administered postoperatively. Animals were sacrificed by $\mathrm{CO}_{2}$ inhalation either 1 (group $1 n=4$; group $2 n=4$ ) or 3 months (group $1 n=3$; group $2 n=5$ ) after implantation. The implants were recovered and each sample was divided into two pieces: one was fixed with $4 \%$ glutaraldehyde in $0.1 \mathrm{M}$ cacodylate buffer for SEM and processed as described above and one was fixed in $10 \%$ neutral buffered formalin and paraffin-embedded. Five $\mu \mathrm{m}$ thick sections were treated with Movat pentachromic stain kit according to the manufacturer's instruction. This stain produces purple-black elastic fibers and nuclei, blue to green mucins, red muscle, and red fibrinoid against a yellow background with collagen.

\section{Results and Discussion}

The ideal biomaterial for the generation of TEBVs should not only possess the mechanical properties of the native vessels but also promote in vivo a regenerative response through the induction of host cell ingrowth. In this context, AMs, obtained by a detergent-enzymatic treatment, have been proven to support in vitro adhesion, growth, and function of several cell types [20-22]. Furthermore, the decellularization process induces the loss of the major histocompatibility complex markers but maintains angiogenic factors, such as b-FGF and TGF- $\beta[13,23,24]$. Thus, AMs can present angiogenic activity, an important factor for the in vivo integration of the tissue substitutes. Indeed, in vivo AMs act as a template allowing the host cell ingrowth and they are remodeled in a living tissue [25, 25-27]. Moreover, they represent preformed structures whose length and gauges can be chosen according to the dimension of the segment to be repaired. Another advantage is the possibility to have easy and unlimited availability of inexpensive grafts containing tissue-specific proteins. Herein, two cycles of detergentenzymatic treatment were needed to completely remove cells from iliac arteries (Figure 1). To detach the endothelial layer (Figure 1(a)), still present at the end of the first cycle, a treatment with collagenase IV was needed between the two decellularization cycles (Figure $1(\mathrm{~d})$ ). The structure of the native vessels was well preserved in AMs that lacked both MHC I (Figure 1(e)) and II (Figure 1(f)) cell membrane antigens, normally present in native tissue (Figures 1(b) and 1(c)).

To guarantee an in vivo long-term patency of TEBVs, intimal hyperplasia and graft occlusion must be avoided. To achieve this goal, a continuous lining of ECs on the luminal surface of TEBVs seems to be essential since it represents a physical barrier that is able to prevent platelet adhesion and the activation of the coagulation cascade [28]. Furthermore, cell seeding reduces the overall influx of macrophages and the magnitude of M1 activation, avoiding scar formation [29]. These effects, in turn, lead to a functional remodeling of the vessel wall. In particular, elastic fibers play a pivotal role as they determine the mechanical properties of both high and small resistance vessels, thus preventing stenosis [30].

Autologous ECs harvested from blood vessel, such as veins, are terminally differentiated, have limited proliferation potential, and lose their function during in vitro expansion [31]. Alternatively, bone marrow-derived mononuclear mesenchymal stromal cells (BM-MSCs) [32] and endothelial progenitor cells (EPCs) $[33,34]$ have been used to make grafts hemocompatible. Nevertheless, the use of these cells can be limited by their low number in the adult tissues, low proliferative rate, and the invasive procedures needed to obtain them, leading to morbidity for the donors. Furthermore, BM-MSCs may induce calcification and thrombus formation [35]. In this work, microvascular ECs were obtained from skin biopsy; that is, a moderately invasive procedure that avoids the need to remove healthy vessels. ECs presented a polygonal shape (Figure 2(a)) and maintained the endothelial phenotype till the fourth culture passage as demonstrated by the expression of von Willebrand factor (Figure 2(b)). Furthermore, at $72 \mathrm{~h}$ from seeding onto AMs, they formed an almost continuous monolayer on the luminal surface of AMs (Figures 2(c) and $2(\mathrm{~d})$ ) and maintained their immunoreactivity to antiCD31 antibody (data not shown). Notably, starting from a $1 \mathrm{~cm}^{2}$ skin fragment, $6 \times 10^{6} \mathrm{ECs}$ were obtained in about 10 days, allowing the production of an implantable graft within 


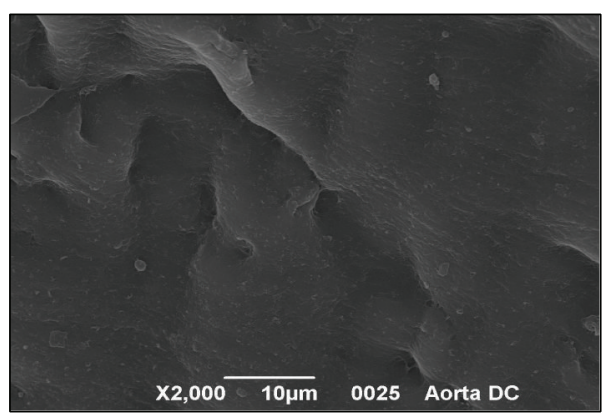

(a)

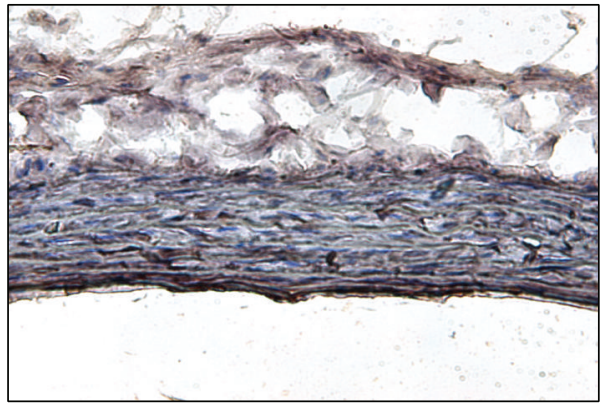

(b)

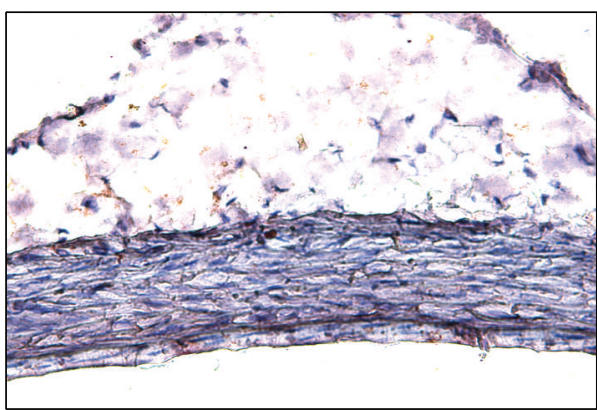

(c)

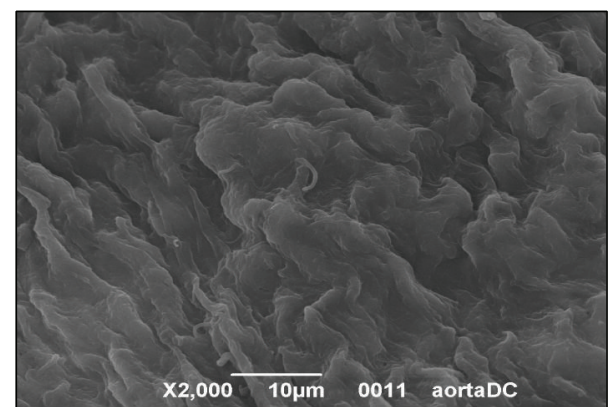

(d)

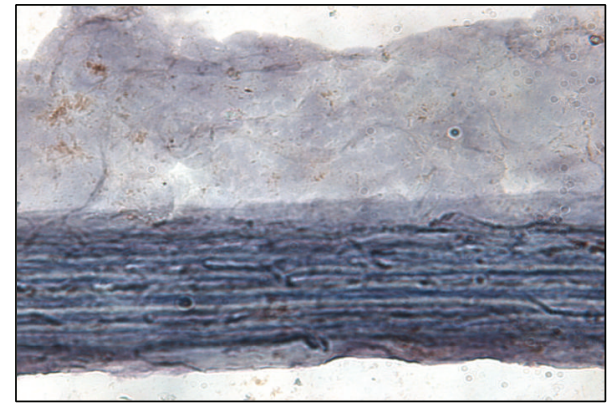

(e)

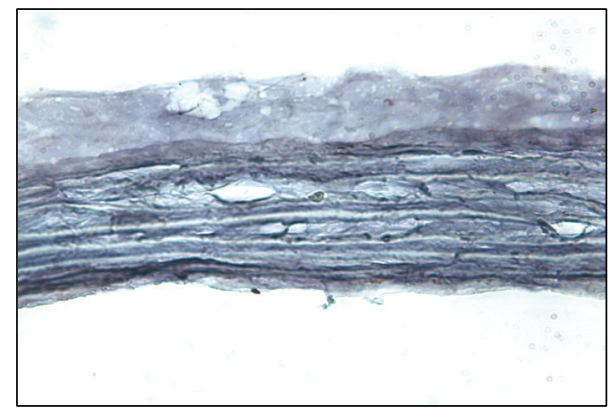

(f)

FIGURE 1: Iliac arteries before $(a-c)$ and after (d-f) the decellularization treatment. (a), (d) SEM micrographs of the luminal sides. Immunoreactivity against MHC I (b, e) and II (c, f) antigens stains brown (magnification $\times 200$ ).

less than two weeks. This period may represent a clinically relevant time frame. Other vascular regeneration techniques already used in clinical practice take about 4-6 weeks to generate TEBVs [4].

As expected, the implantation of only AMs into the abdominal aorta of female Lewis rats gave unsatisfactory results. Although all animals survived, at both 1 and 3 months (Figures 3(a) and 3(b)), explanted grafts presented higher external diameters than those of host vessels. One month after surgery, only the borders of the patch were reendothelialized (Figure 4(a)), whereas, in the other areas, several platelets adhered to the exposed collagen fibers (Figure 4(b)). Only one animal did not present thrombi inside the implanted AM. At 3 months, the luminal surface was almost completely covered by ECs (Figures 4(c) and 4(d)) and no thrombi were detected. At both time points, although elastic fibers were well organized, neointimal hyperplasia and thickening of the adventitial layer were evident (Figures 5(a) and 5(b)). Furthermore, the adventitia of implanted AM grafts presented a more fibrous structure than that observed in the native aorta (Figure 5(e)). The latter evidence agrees with the findings of Assmann et al. [36], who transplanted homologous decellularized aortic conduits in the infrarenal aorta of rats. Although the grafts remained patent for 8 weeks, hyperplastic tissue formation and implant microcalcification occurred. Neointimal hyperplasia was also detected in decellularized and heparinized grafts implanted in dogs as carotid artery bypass grafts [16].

The in vitro coverage of the luminal side of AMs with skin microvascular ECs greatly improved the outcomes of the reconstructive surgery avoiding thrombus formation and allowing good patency. It has been already demonstrated that autologous cells, rather than to mask platelets adhesion sites, may produce soluble factors that are able to enroll the cells 


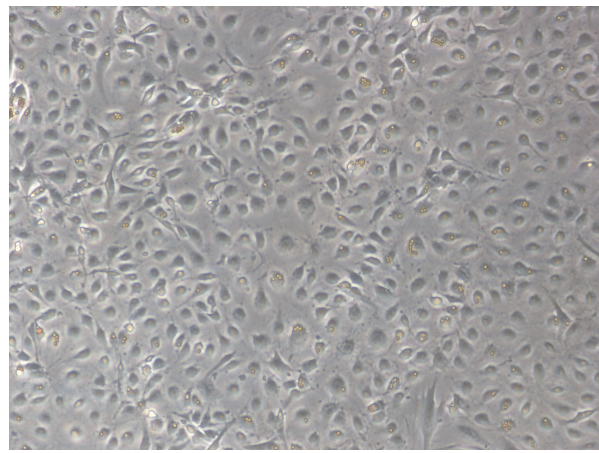

(a)

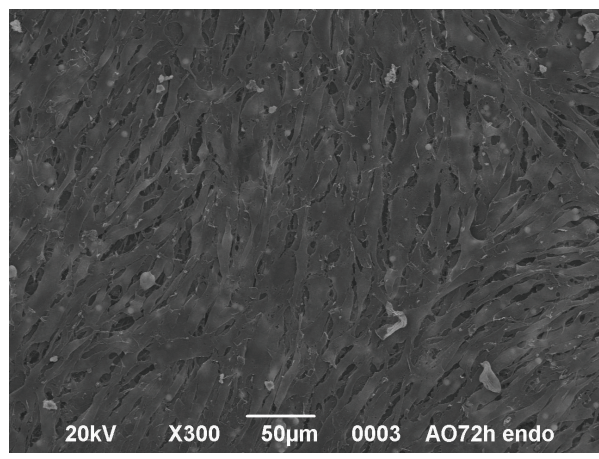

(c)

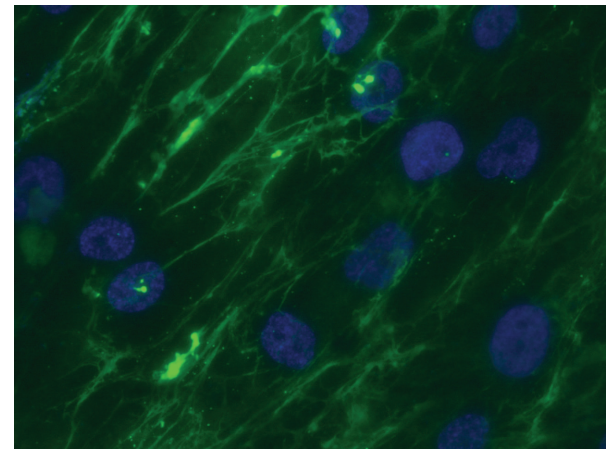

(b)

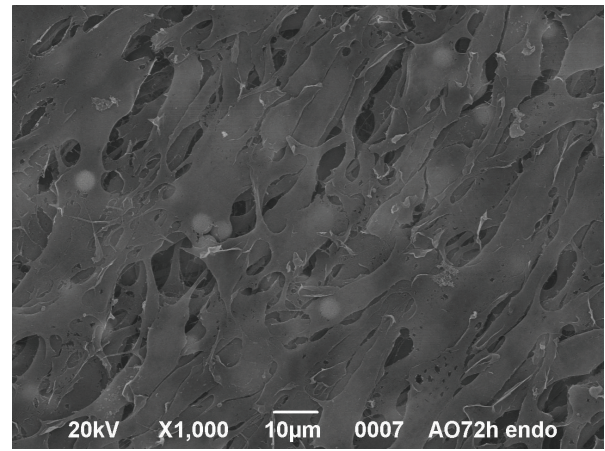

(d)

Figure 2: Cultures of skin microvascular ECs on tissue culture treated plates (a, b) and AMs (c, d). (a) Phase-contrast microscopy (magnification $\times 100$ ). (b) Immunofluorescence carried out using anti-von Willebrand factor antibody (magnification $\times 400)$. (c), (d) SEM micrographs of ECs/AM cultures at $72 \mathrm{~h}$ from seeding.

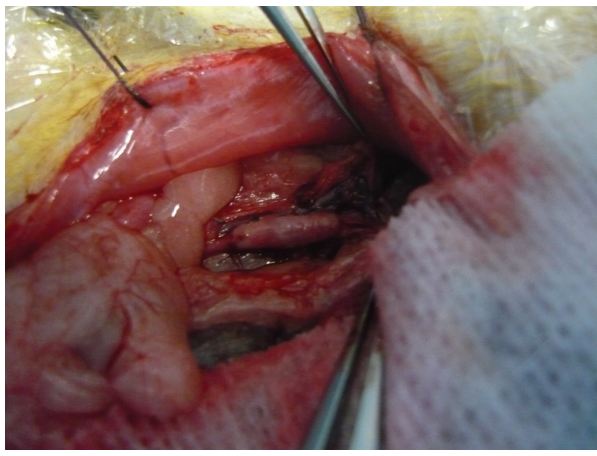

(a)

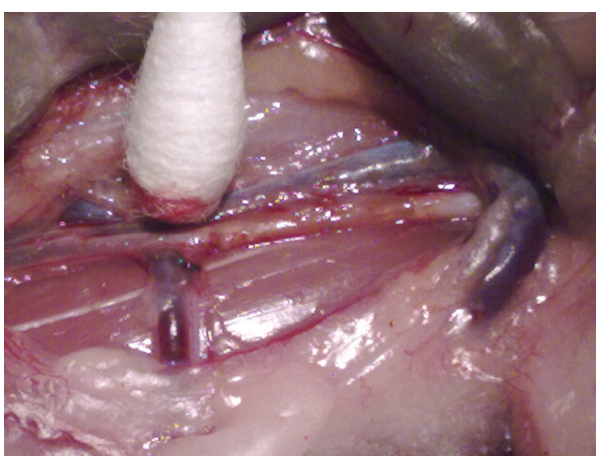

(c)

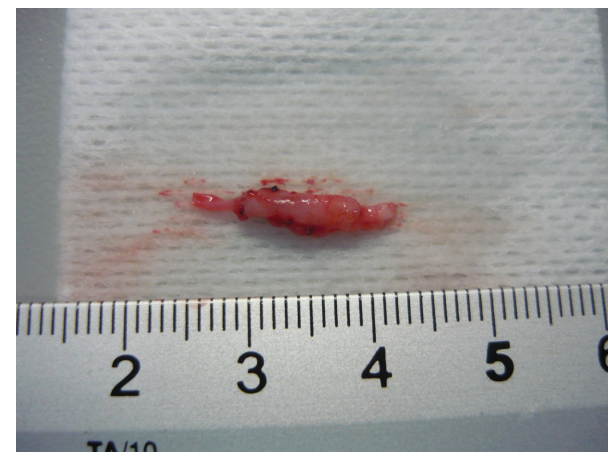

(b)

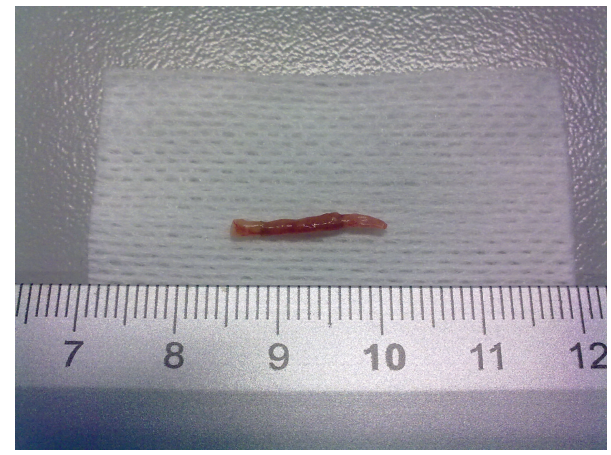

(d)

FIgURE 3: Implants composed of AMs (a, b) and ECs and AMs (c, d) 3 months after surgery. 


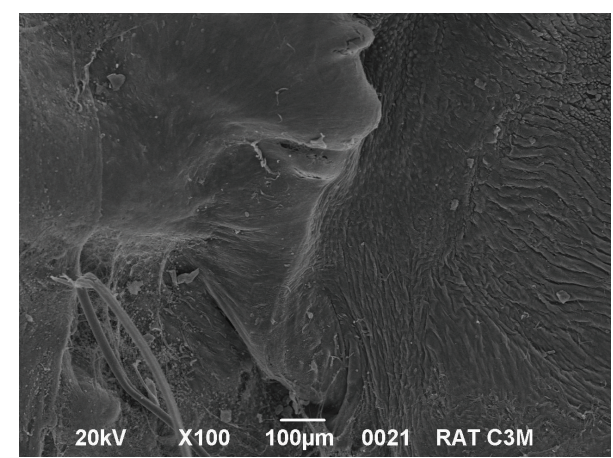

(a)

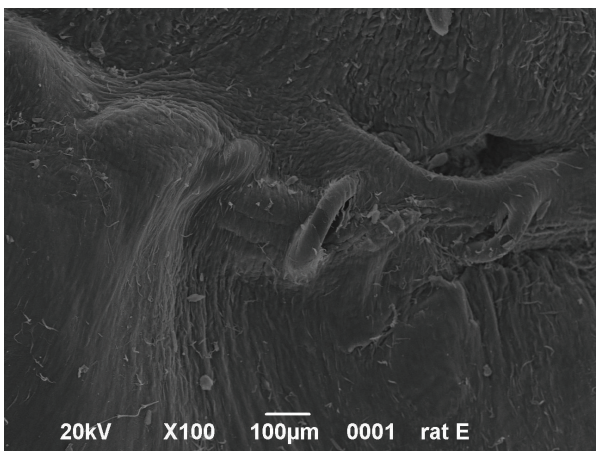

(c)

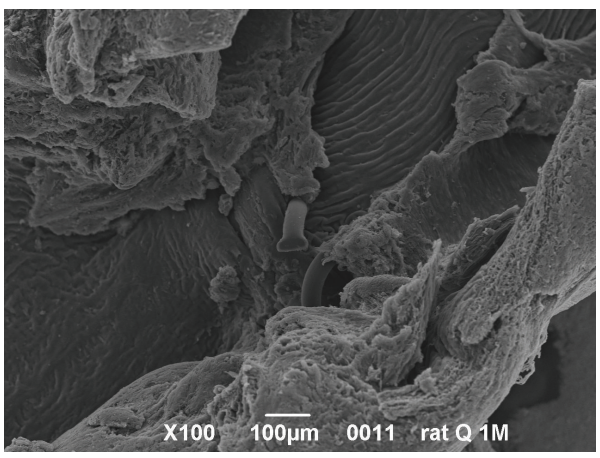

(e)

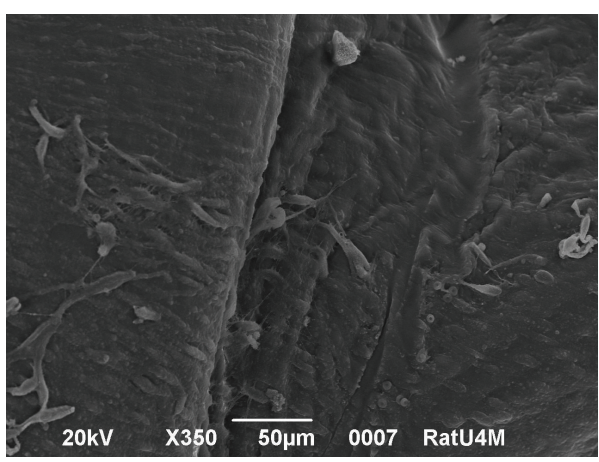

(g)

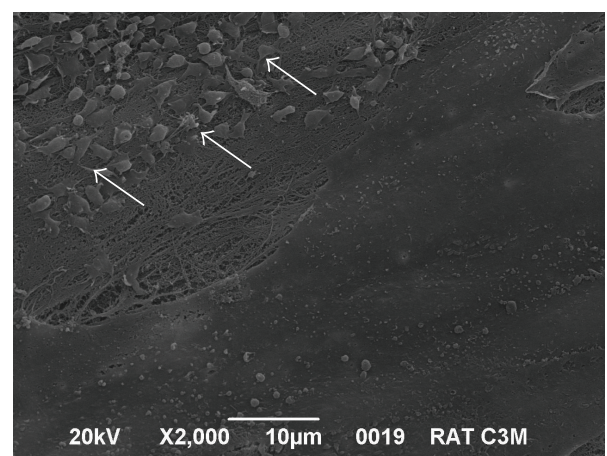

(b)

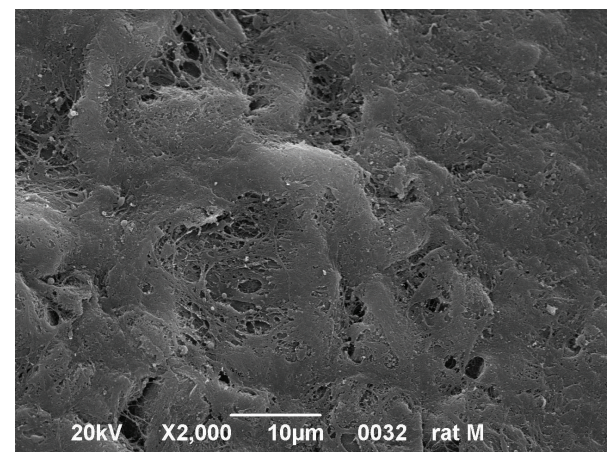

(d)

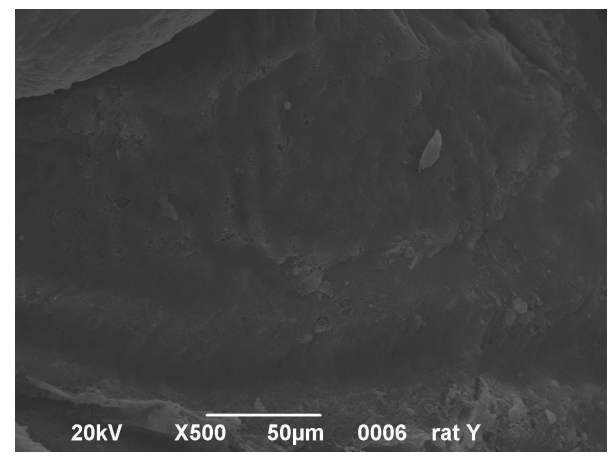

(f)

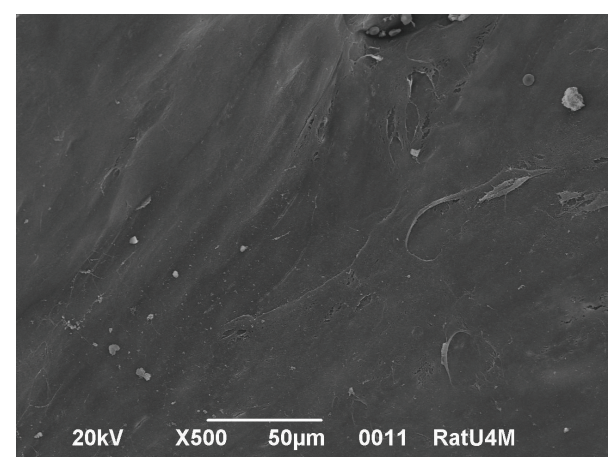

(h)

FIGURE 4: SEM micrographs of the luminal sides of AMs (a-d) and ECs/AM grafts (e-h) at 1 (a, b, e, f) and 3 (c, d, g, h) months after surgery. Arrows indicate area covered by platelets. 


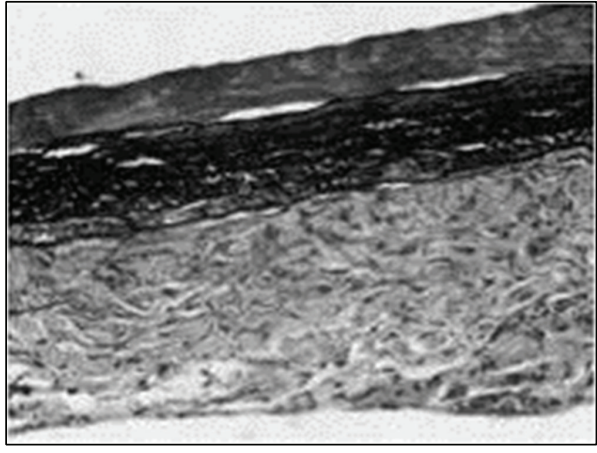

(a)

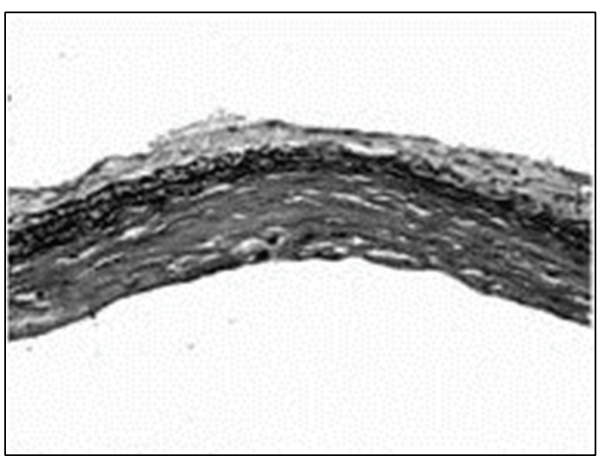

(c)

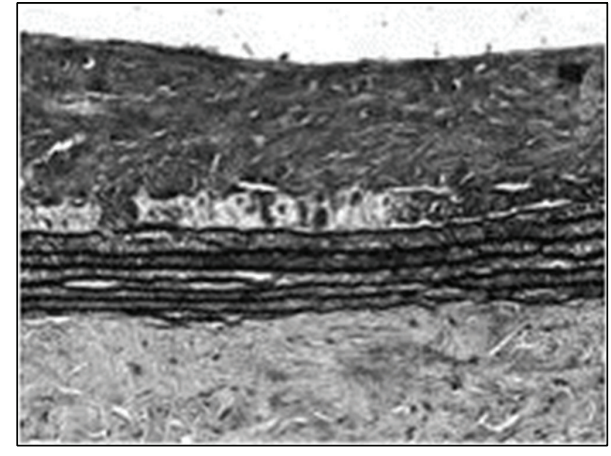

(b)

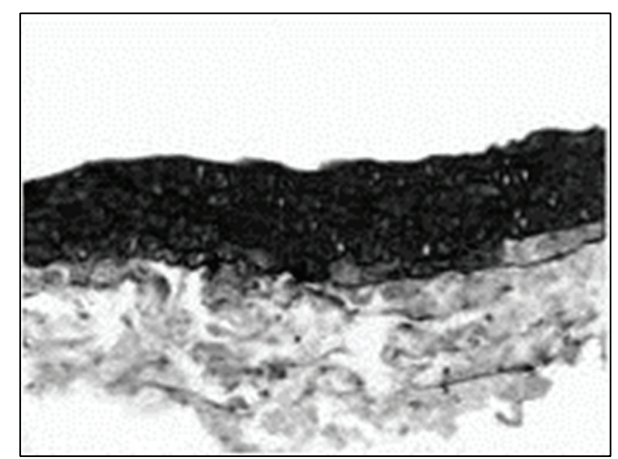

(d)

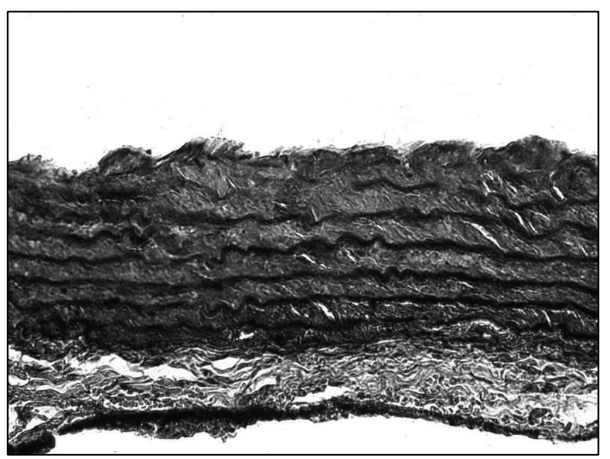

(e)

Figure 5: Movat staining of AMs (a, b) and ECs/AM (c, d) grafts after $1(a-c)$ and $3(b-d)$ months from surgery (magnification $\times 200)$. (e) Native aorta.

from the neighbouring host tissues [37]. Furthermore, the seeded cells may lead to the homing of circulating monocytes [29] that, in turn, release the monocyte chemotactic protein-1, stimulating the regeneration of the blood vessel [38]. Although it has not been verified whether the seeded ECs were present on the implanted grafts, we can suppose that the initial endothelial coverage could be transient and progressively replaced by the host cells [39]. At both time points, the external diameter of the explanted grafts was similar to the one of host aorta (Figures 3(c) and 3(d)). One month from surgery, all luminal surfaces were completely reendothelialized and no signs of platelet adhesion were visible (Figures 4(e)-4(h)). The implanted grafts appeared to be remodelled during the time (Figures 5(c) and 5(d)). Indeed, at 1 month, histological analysis revealed a moderate hyperplasia of the tunica intima that disappeared at 3 months. Furthermore, the thickness of the elastic layer increased over the time. Similar results were obtained by Leyh et al. [40] who implanted TEBVs composed of homologous decellularized arteries with or without autologous ECs in the pulmonary circulation of sheep. The in vitro reendothelialized conduits performed well for as long as 6 months, whereas the lack of ECs led to aneurysm formation.

\section{Conclusions}

Herein, we show that AM-based TEBVs lead to unsatisfactory results since the lack of ECs contributes to graft thrombogenicity and promotes intimal proliferation. One month after surgery, the luminal surface of implanted AMs was partially 
covered by ECs and several platelets adhered in the areas lacking cell coverage. Intimal hyperplasia, already detected after 1 month, increased at 3 months. On the contrary, the in vitro reendothelialized AMs led to well-performing vascular conduits whose structure resembles that of the host vessel. The ECs lining the luminal surface of the grafts function as a barrier preventing the platelet adhesion on extracellular matrix, and they may influence, in a paracrine manner, the regeneration process by recruiting the host cells and modulating the inflammatory response. In agreement with this statement, our results demonstrated that only the implanted ECs seeded grafts were progressively changed with a thickening of the tunica media. Thus, the cellular component of the graft allows AMs to act as a temporary template that can be correctly remodelled by the host cells in a functional tissue.

\section{Conflict of Interests}

The authors declare that there is no conflict of interests regarding the publication of this paper.

\section{Authors' Contribution}

Luigi Dall'Olmo and Ilenia Zanusso equally contributed to this paper and should be considered co-first authors.

\section{Acknowledgments}

This research was supported by Regione Veneto (Italy). The authors thank Mr. Mariano Schiavon for the laboratory assistance.

\section{References}

[1] A. T. Hirsch, M. A. Allison, A. S. Gomes et al., "A call to action: women and peripheral artery disease: a scientific statement from the American Heart Association," Circulation, vol. 125, no. 11, pp. 1449-1472, 2012.

[2] J. G. Nemeno-Guanzon, S. Lee, J. R. Berg et al., "Trends in tissue engineering for blood vessels," Journal of Biomedicine and Biotechnology, vol. 2012, Article ID 956345, 2012.

[3] V. Piccone, "Alternative techniques in coronary artery reconstruction," in Modern Vascular Grafts, P. N. Sawyer, Ed., pp. 253260, McGraw-Hill, New York, NY, USA, 1987.

[4] L. P. Neff, B. W. Tillman, S. K. Yazdani et al., "Vascular smooth muscle enhances functionality of tissue-engineered blood vessels in vivo," Journal of Vascular Surgery, vol. 53, no. 2, pp. 426-434, 2011.

[5] A. C. Thomas, G. R. Campbell, and J. H. Campbell, "Advances in vascular tissue engineering," Cardiovascular Pathology, vol. 12, no. 5, pp. 271-276, 2003.

[6] H. S. Hung, H. C. Chen, C. H. Tsai, and S. Z. Lin, "Novel approach by nanobiomaterials in vascular tissue engineering," Cell Transplantation, vol. 20, no. 1, pp. 63-70, 2011.

[7] M. T. Conconi, L. Borgio, R. Di Liddo et al., "Evaluation of vascular graft based on polyvinyl alcohol cryogels," Molecular Medicine Reports, 2014.

[8] T. Shin'oka, G. Matsumura, N. Hibino et al., "Midterm clinical result of tissue-engineered vascular autografts seeded with autologous bone marrow cells," The Journal of Thoracic and Cardiovascular Surgery, vol. 29, pp. 1330-1338, 2005.

[9] N. L'Heureux, S. Pâquet, R. Labbé, L. Germain, and F. A. Auger, "A completely biological tissue-engineered human blood vessel," The FASEB Journal, vol. 12, no. 1, pp. 47-56, 1998.

[10] N. L'Heureux, S. Paquet, R. Labbe, L. Germain, and F. A. Auger, "Tissue-engineered blood vessel for adult arterial revascularization," The New England Journal of Medicine, vol. 357, pp. 14511453, 2007.

[11] T. N. McAllister, M. Maruszewski, S. A. Garrido et al., "Effectiveness of haemodialysis access with an autologous tissueengineered vascular graft: a multicentre cohort study," The Lancet, vol. 373, no. 9673, pp. 1440-1446, 2009.

[12] T. W. Gilbert, T. L. Sellaro, and S. F. Badylak, "Decellularization of tissues and organs," Biomaterials, vol. 27, no. 19, pp. 36753683, 2006.

[13] M. T. Conconi, B. Nico, D. Mangieri et al., "Angiogenic response induced by acellular aortic matrix in vivo," Anatomical Record A: Discoveries in Molecular, Cellular, and Evolutionary Biology, vol. 281, no. 2, pp. 1303-1307, 2004.

[14] J. P. Hodde, R. D. Record, H. A. Liang, and S. F. Badylak, "Vascular endothelial growth factor in porcine-derived extracellular matrix," Endothelium, vol. 8, no. 1, pp. 11-24, 2001.

[15] D. R. Clarke, R. M. Lust, Y. S. Sun, K. S. Black, and J. D. Ollerenshaw, "Transformation of nonvascular acellular tissue matrices into durable vascular conduits," Annals of Thoracic Surgery, vol. 71, no. 5, pp. S433-S436, 2001.

[16] B. S. Conklin, E. R. Richter, K. L. Kreutziger, D.-S. Zhong, and C. Chen, "Development and evaluation of a novel decellularized vascular xenograft," Medical Engineering and Physics, vol. 24, no. 3, pp. 173-183, 2002.

[17] J. I. Spark, S. Yeluri, C. Derham, Y. T. Wong, and D. Leitch, "Incomplete cellular depopulation may explain the high failure rate of bovine ureteric grafts," British Journal of Surgery, vol. 95, no. 5, pp. 582-585, 2008.

[18] E. Meezan, J. T. Hjelle, K. Brendel, and E. C. Carlson, "A simple, versatile, nondisruptive method for the isolation of morphologically and chemically pure basement membranes from several tissues," Life Sciences, vol. 17, no. 11, pp. 1721-1732, 1975.

[19] M. P. Pusztaszeri, W. Seelentag, and F. T. Bosman, "Immunohistochemical expression of endothelial markers CD31, CD34, von Willebrand factor, and Fli-1 in normal human tissues," Journal of Histochemistry and Cytochemistry, vol. 54, no. 4, pp. 385-395, 2006.

[20] P. Burra, S. Tomat, M. T. Conconi et al., "Acellular liver matrix improves the survival and functions of isolated rat hepatocytes cultured in vitro," International journal of molecular medicine, vol. 14, no. 4, pp. 511-515, 2004.

[21] M. Dettin, M. T. Conconi, R. Gambaretto et al., "Effect of synthetic peptides on osteoblast adhesion," Biomaterials, vol. 26, no. 22, pp. 4507-4515, 2005.

[22] M. T. Conconi, P. de Coppi, R. di Liddo et al., "Tracheal matrices, obtained by a detergent-enzymatic method, support in vitro the adhesion of chondrocytes and tracheal epithelial cells," Transplant International, vol. 18, no. 6, pp. 727-734, 2005.

[23] D. Ribatti, M. T. Conconi, B. Nico et al., "Angiogenic response induced by acellular brain scaffolds grafted onto the chick embryo chorioallantoic membrane," Brain Research, vol. 989, no. 1, pp. 9-15, 2003. 
[24] M. T. Coconi, B. Nico, P. Rebuffat et al., "Angiogenic response induced by acellular femoral matrix in vivo," Journal of Anatomy, vol. 207, no. 1, pp. 79-83, 2005.

[25] M. Marzaro, S. Vigolo, B. Oselladore et al., "In vitro and in vivo proposal of an artificial esophagus," Journal of Biomedical Materials Research A, vol. 77, no. 4, pp. 795-801, 2006.

[26] M. T. Conconi, F. Rocco, R. Spinazzi et al., "Biological fate of tissue-engineered porcine valvular conduits xenotransplanted in the sheep thoracic aorta," International Journal of Molecular Medicine, vol. 14, no. 6, pp. 1043-1048, 2004.

[27] P. Macchiarini, P. Jungebluth, T. Go et al., "Clinical transplantation of a tissue-engineered airway," The Lancet, vol. 372, no. 9655, pp. 2023-2030, 2008.

[28] D. Pankajakshan and D. K. Agrawal, "Scaffolds in tissue engineering of blood vessels," Canadian Journal of Physiology and Pharmacology, vol. 88, no. 9, pp. 855-873, 2010.

[29] N. Hibino, T. Yi, D. R. Duncan et al., "A critical role for macrophages in neovessel formation and the development of stenosis in tissue-engineered vascular grafts," The FASEB Journal, vol. 25, no. 12, pp. 4253-4263, 2011.

[30] J. Ferruzzi, M. J. Collins, A. T. Yeh, and J. D. Humphrey, "Mechanical assessment of elastin integrity in fibrillin-1deficient carotid arteries: implications for Marfan syndrome," Cardiovascular Research, vol. 92, no. 2, pp. 287-295, 2011.

[31] G. Grenier, M. Rémy-Zolghadri, R. Guignard et al., "Isolation and culture of the three vascular cell types from a small vein biopsy sample," In Vitro Cellular \& Developmental BiologyAnimal, vol. 39, no. 3-4, pp. 131-139, 2003.

[32] Y. Zhao, S. Zhang, J. Zhou et al., "The development of a tissueengineered artery using decellularized scaffold and autologous ovine mesenchymal stem cells," Biomaterials, vol. 31, no. 2, pp. 296-307, 2010.

[33] B. W. Tillman, S. K. Yazdani, L. P. Neff et al., "Bioengineered vascular access maintains structural integrity in response to arteriovenous flow and repeated needle puncture," Journal of Vascular Surgery, vol. 56, no. 3, pp. 783-793, 2012.

[34] M. Zhou, Z. Liu, C. Liu et al., "Tissue engineering of smalldiameter vascular grafts by endothelial progenitor cells seeding heparin-coated decellularized scaffolds," Journal of Biomedical Materials Research B: Applied Biomaterials, vol. 100, no. 1, pp. 111-120, 2012.

[35] Y. Yoon, J. Park, T. Tkebuchava, C. Luedeman, and D. W. Losordo, "Unexpected severe calcification after transplantation of bone marrow cells in acute myocardial infarction," Circulation, vol. 109, no. 25, pp. 3154-3157, 2004.

[36] A. Assmann, P. Akhyari, C. Delfs et al., "Development of a growing rat model for the in vivo assessment of engineered aortic conduits," Journal of Surgical Research, vol. 176, no. 2, pp. 367-375, 2012.

[37] J. Y. Liu, D. D. Swartz, H. F. Peng, S. F. Gugino, J. A. Russell, and S. T. Andreadis, "Functional tissue-engineered blood vessels from bone marrow progenitor cells," Cardiovascular Research, vol. 75, no. 3, pp. 618-628, 2007.

[38] J. D. Roha, R. Sawh-Martinez, M. P. Brennan et al., “Tissueengineered vascular grafts transform into mature blood vessels via an inflammation-mediated process of vascular remodeling," Proceedings of the National Academy of Sciences of the United States of America, vol. 107, no. 10, pp. 4669-4674, 2010.

[39] M. A. Cleary, E. Geiger, C. Grady, C. Best, Y. Naito, and C. Breuer, "Vascular tissue engineering: the next generation," Trends in Molecular Medicine, vol. 18, no. 7, pp. 394-404, 2012.
[40] R. G. Leyh, M. Wilhelmi, P. Rebe, S. Ciboutari, A. Haverich, and H. Mertsching, "Tissue engineering of viable pulmonary arteries for surgical correction of congenital heart defects," Annals of Thoracic Surgery, vol. 81, no. 4, pp. 1466-1471, 2006. 


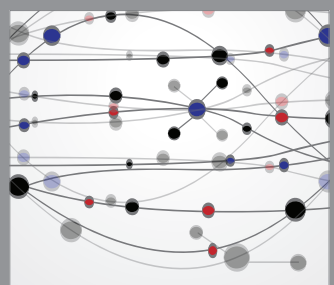

The Scientific World Journal
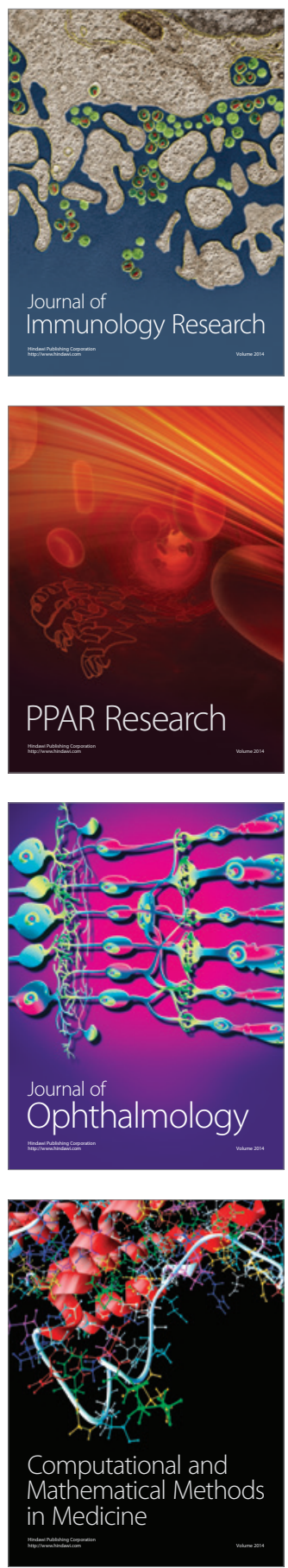

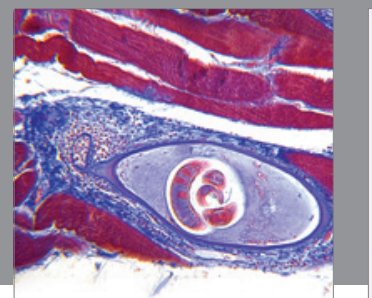

Gastroenterology

Research and Practice
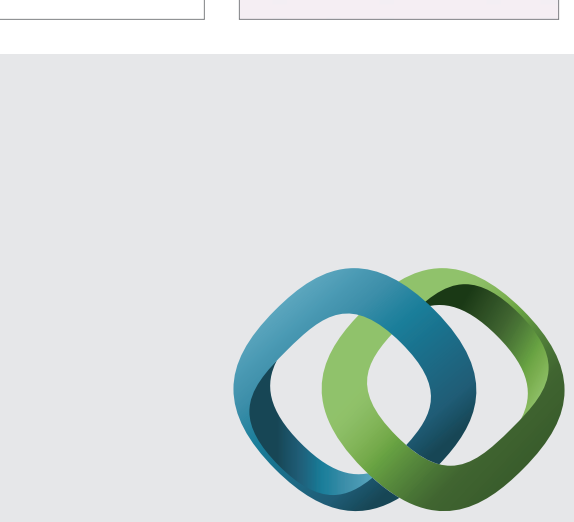

\section{Hindawi}

Submit your manuscripts at

http://www.hindawi.com
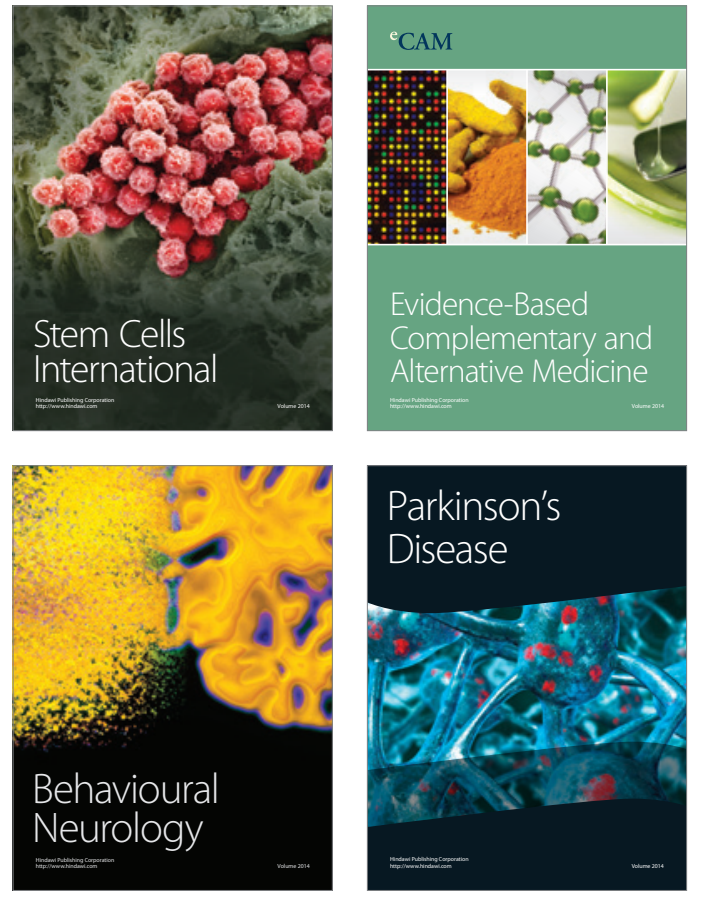
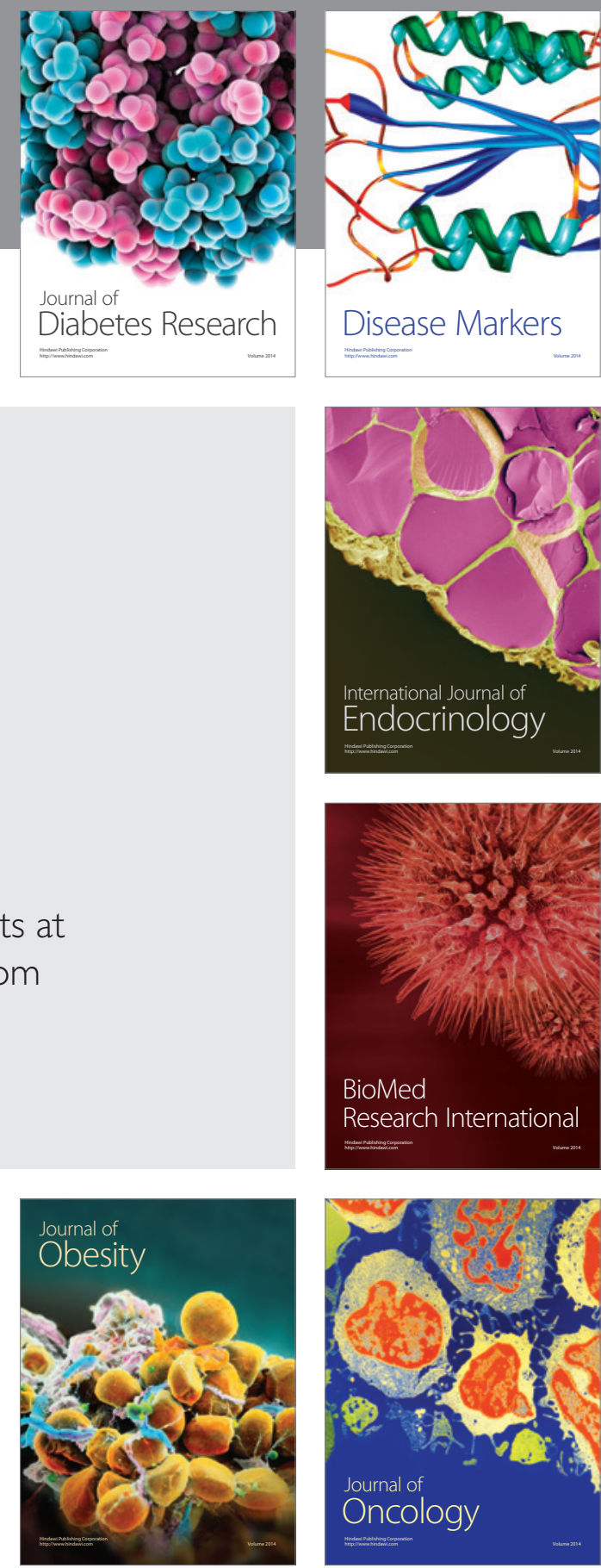

Disease Markers
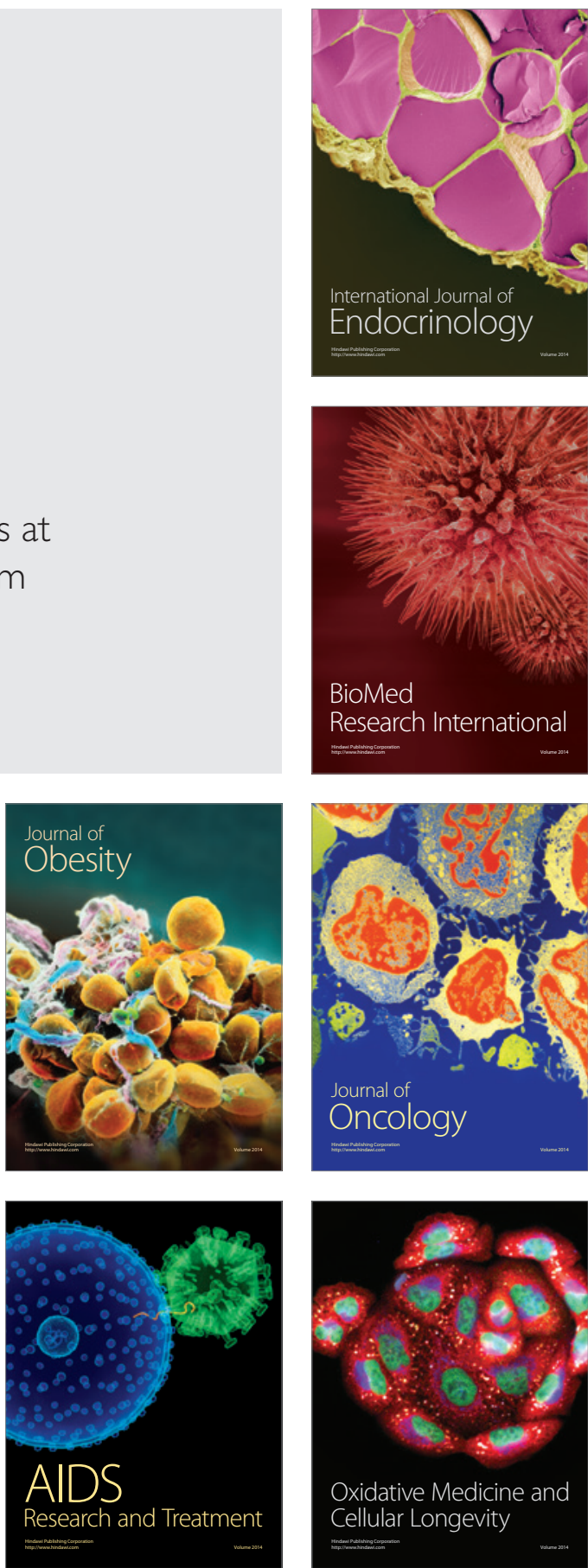